\title{
Long Bone Panoramas from Fluoroscopic X-ray Images
}

\author{
Z. Yaniv and L. Joskowicz \\ School of Computer Science and Engineering \\ The Hebrew University of Jerusalem, Jerusalem 91904 Israel. \\ zzivy,josko\}@cs.huji.ac.il
}

We have developed a new method for creating a single panoramic image of a long bone from a few individual fluoroscopic X-ray images [1]. Fluoroscopic X-ray panoramas can be useful in a variety of orthopaedic surgeries which require the presence in the same image of relevant anatomical features. In long bone surgery, they can be used for determining the mechanical axis of the bone, aligning bone fragments, measuring extremity length and anteversion, and assessing long implants positions. This data is difficult to obtain with existing methods and can help to improve diagnosis, shorten surgery time, and improve outcomes.

Our method uses a radiolucent ruler imaged alongside the long bone to establish the correlation between images. Before the surgery, an aluminum grid is imaged and a distortion correction map is computed [2]. Then, the radiolucent ruler is placed roughly parallel and at the same height of the long bone to be imaged. Several overlapping images (between $20 \%$ and $60 \%$ of their area) are taken by translating the fluoroscope parallel to the bone. The individual images are corrected for distortion, and the main thread and graduations of the ruler are extracted from the images using two-value histogram thresholding. The ruler graduations are matched using cross correlation as a similarity measure, and the rigid transformation that aligns them is computed. The undistorted images are then aligned according to the these transformations, and then composed into a single image by computing pixel values at locations where images overlap.

Our experiments show the method is robust and produces panoramas with an accuracy comparable to that of individual images, and are thus clinically acceptable. The advantages of our method are that it is fully automatic, uses readily available hardware, requires a simple image acquisition protocol and minimal user intervention, and works with most existing fluoroscopic units without modifications. 1

1. Yaniv, Z. and Joskowicz, L., "Long bone panoramas from fluoroscopic X-ray images", Proc. of the 15th Int. Congress on Computer-Assisted Radiology and Surgery, CARS'2001, H.U. Lemke et. al. eds, Elsevier 2001.

2. Yaniv Z., Joskowicz L., Simkin A., Garza-Jinich M., Milgrom C. "Fluoroscopic Image Processing for Computer-Aided Orthopaedic Surgery", 1st Int.

\footnotetext{
${ }^{1}$ This research was supported in part by a grant from the Israel Ministry of Industry and Trade for the IZMEL Consortium on Image-Guided Therapy.
} 
Conf. on Medical Computing and Computer-Assisted Intervention, Lecture

Notes in Computer Science 1496, Elsevier, Wells et. al. eds, 1998.

\section{Long bone panorama construction}
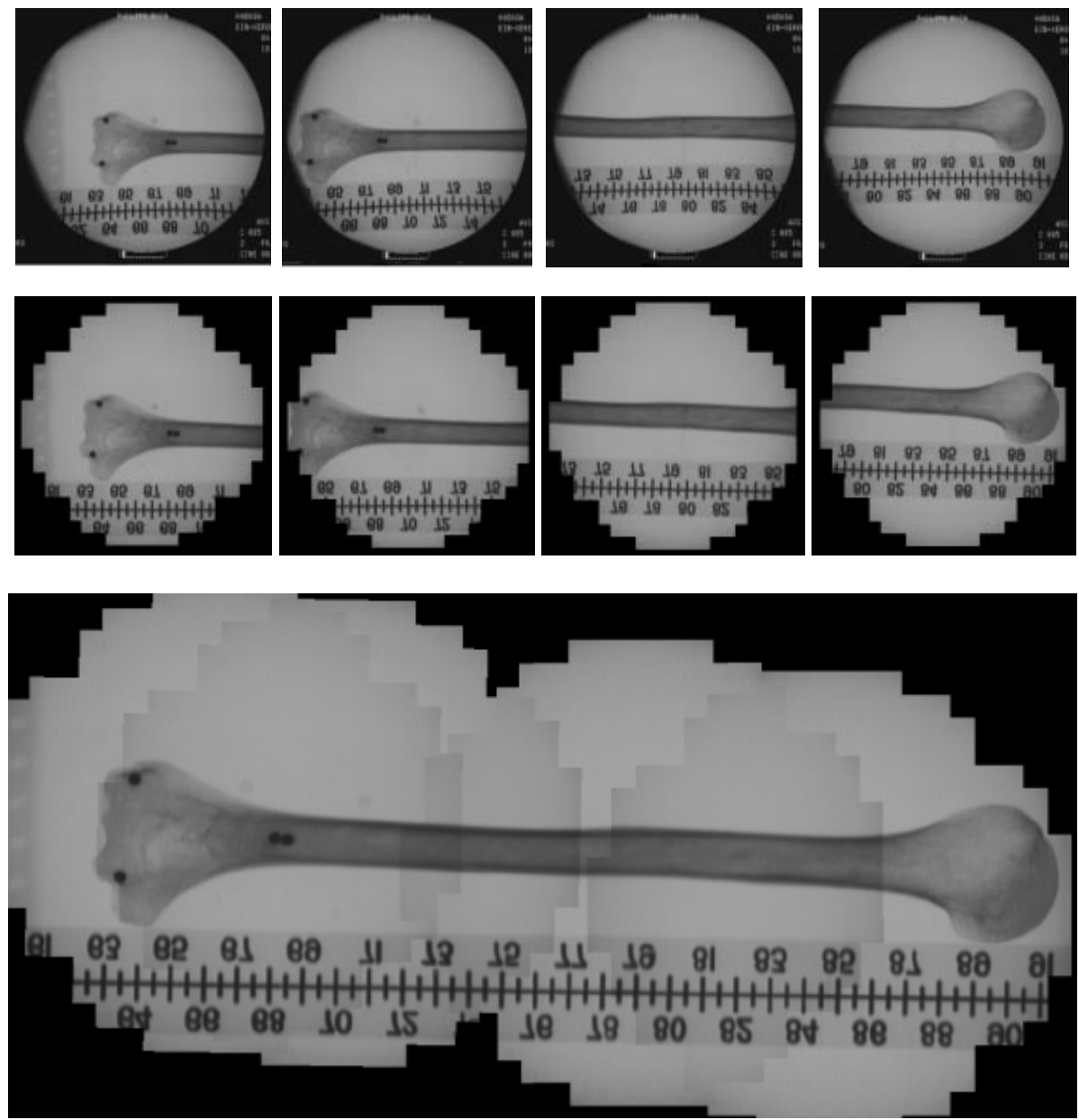

Fig. 1. Panorama construction of a dry humerus. The top row shows the original images with the ruler on the bottom, the middle row shows the images after distortion correction, and the bottom row shows the resulting panorama. 